\title{
Über die Aufnahme lipoidlöslicher Kohlenwasserstoffe als Indikator für die Durchblutung von Fettgewebe und über die Steigerung ihrer Toxizität bei Leberverfettung
}

\author{
Von J. G. Gostomzy K \\ Aus dem Department für Anaestbesiologie (Leiter: Prof. Dr. F. W. Abnefeld und Prof. Dr. W. Dick) \\ des Zentrums für Operative Mediqin der Universität Ulm und dem Institut für Recbtsmedizin \\ (Leiter: Prof. Dr. H. Leitboff) der Universität Mainz
}

(Eingegangen am 15. Mai/8. August 1972)

\begin{abstract}
Die Pharmakokinetik flüchtiger lipoidlöslicher Kohlenwasserstoffe in Blut und Gewebe wurde unter Verwendung des Narkosegases Halothan als Modellsubstanz mit Hilfe der Dampfraum-Gaschromatographie untersucht. Die Geschwindigkcit der Anflutung und Elimination des Gases im Depotfett (epididymaler Fettkörper) und im braunen Fettgewebe (interscapulärer Fettkörper) der Ratte ist sehr verschieden, offenbar in Abhängigkeit vom Durchblutungsgrad der Gewebe. Bei Mäusen mit genetisch bedingter Adipositas (homocygote Merkmalträgcr) führt die Verlängerung der Diffusionsstrecke bei Volumenzunahme der Fettzelle und die verminderte Durchblutung zu einem langsameren Konzentrationsanstieg der Gase im epididymalen Fettkörper. Die Einbaurate in das braune Fettgewebs dieser Tiere ist dagegen ebenso groß wie bei NMRI-Mäusen.

Die Leberzelle der adipösen Mäuse ist verfettet. Die pathologischen Fettablagerungen führen unter gleichen Expositionsbedingungen zu einer gegenüber normalen NMRI-Mäusen 4-fach höheren Gaskonzentration im Lebergewebe adipöser Tiere, wobei es nach Mehrfachexposition zu einer lichtmikroskopisch nachweisbaren Zunahme der Verfettung kommt. Die Bedeutung der Befunde für die pränarkotische Leberdiagnostik, für die Begutachtung von Intoxikationen mit lipoidlöslichen Gasen und für die arbeitsmedizinische Beurteilung der MAK-Werte wird diskutiert.
\end{abstract}

\section{On the uptake of lipid-soluble bydrocarbons as an indicator for the blood circulation in fat tissues, and on the increase in their toxicity' in fatty livers}

The pharmacokinetics of volatile, lipid-soluble hydrocarbons in blood and tissues were studied, using the anesthetic gas Halothan as a model substance, with the aid of vapor phase gas chromatography. The rates of uptake and elimination of the gases in the depot fat (epididymal fat pads) and in the brown fat tissue (interscapular fat pads) of the rat are very different, apparently depending on the amount of blood circulation in the tissue. In mice with genetically caused adipositas (homozygotic gene carriers), the reduced diffusion rate, caused by the increased volume of the fat cells and the decreased blood circulation, lead to a slower concentration buildup of the gases in the epididymal fat pads. The buildup rate in the brown fat tissues of these animals is, in contrast, just as great as in NMRI mice.

The liver cell of an adipose mouse is fatty. The pathological fat storage leads, under identical exposure conditions, to a 4-fold higher gas concentration in the liver tissues of adipose animals than in the livers of normal NMRI mice. Further, after repeated exposure, an increase in the fat storage can be observed with a light microscope. The significance of the findings for the pre-anesthetic liver diagnosis and for the evaluation of intoxications with lipid-soluble gases is discussed. The results are also significant for the evaluation by industrial medicine of the maximum allowable concentration in the working environment.

Inhalierte flüchtige lipoidlösliche Kohlenwasserstoffe werden vornehmlich vom Fettgewebe aufgenommen. Unterliegen sie im Gewebe nicht einer Biotransformation, so wird bei geringer Löslichkeit im Blut und bei vergleichsweise hoher Lösungṣkapazität im Fettgewebe die pro Zeiteinheit in diesem Gewebe deponierte Substanzmenge von dessen Durchblutung bestimmt (FICk'sches Prinzip). Bei konstantem Gasanteil in der Atemluft, der nach Ausbildung des Konzentrationsgleichgewichtes Alveolargas/arterielles Blut die Höhe des Blutspiegels bestimmt, spielt das Konzentrationsgefälle Blut/Gewebe bis zum Erreichen der Gewebesättigung nur eine untergeordnete Rolle. Die vorliegende tierexperimentelle Studie zur Pharmakokinetik lipoidlöslicher Gase weist auf erhebliche Unterschiede in der Durchblutung einzelner Fettgewebe hin. Weiterhin wird gezeigt, da $B$ intrazelluläre Fettablagerungen in parenchymatösen Organen die Lösungskapazität der Zelle für lipophile Substanzen offenbar erhöhen.

\section{Material und Methoden}

Für die Untersuchung der Pharmakokinetik lipoidlöslicher flüchtiger Kohlenwasserstoffe sind Narkosegase gut geeignet, da durch Verwendung von Narkosegeräten mit entsprechenden Verdampfern die Herstellung von Gasgemischen konstanter $\mathrm{Zu}-$ sammensetzung ermöglicht wird. In der vorliegenden Untersuchung wurde Halothan ${ }^{1}$ ) verwendet.

Halothanbestimmung im Gewebe

Für Halothanbestimmungen aus geringen Blutmengen hat sich die Dampfraum-Gaschromatographie (head-space-chromatography) unter Verwendung einer wäßr. Lösung von tertiärem Butanol als internen Standard als brauchbar erwiesen (1). Voraussetzung für reproduzierbare quantitative Analysen aus dem Dampfraum über der zu untersuchenden Probe ist die Ausbildung eines konstanten Dampfdruckes in einem vorgegebenen Volumen (Probengläschen) unter isothermen Bedingungen. Seine $\mathrm{Zu}$ sammensetzung muß in quantitativer Relation zu entsprechenden Lösungskomponenten stehen. Nur stark verdünnte Lösungen von Substanzen mit hohem Dampfdruck erfüllen diese Bedingungen im strengen Sinne.

1) 2-Brom-2-clilor-1,1,1-trifuoräthan, Farbwerkc Hoechst. 
Untersuchungen von nichthomogenisietten Gewebsproben von $500 \pm 50 \mathrm{mg}$ bei Rattenversuchen bzw. 200 خ- $20 \mathrm{mg}$ bei Versuchen mit Mäusen mit Hilfe der Dampfraum-Gaschromatographie ergaben ebenfalls reproduzierbare Ergebnisse. In getrennten Versuchsserien fanden sich eine gute Ubereinstimmung zwischen Alkoholbestimmungen in Blut und Gewebe nach der DampfraumGaschromatographie mit Ergebnissen aus Versuchen mit ${ }^{14} \mathrm{C}$ markiertem Athanol (2). Als Vorteil der Dampfraum-Gaschromatographie ist hervorzuheben, $\mathrm{da} B$ die Probe sofort nach Entnahme und Wägung in das verschließbare Analyseglas gebrach wird, so daß Substanzverluste weitgehend vermieden werden.

Die Analysenergebnisse werden als relativer Wert $Q=\frac{h_{P r}}{h_{S t}}$ (hpr $=$ Höhe des Peaks der Probe im Chromatogramm, hst $=$ Höhe des Peaks des internen Standards) angegeben. Die absoluten Konzentrationen $(\mathrm{mg} / \mathrm{l})$ sind vom Dampfdruck über dem Lösungsmittel abhängig, wie Versuche mit Halothan in wäßr. und öliger Lösung zeigen (Abb. 1a und $\mathrm{b}$ ). Aus diesen Versuchen lassen sich folgende mittlere Umrechnungsfaktoren für Analysen aus $0,5 \mathrm{ml}$ Lösung für Konzentrationen bis etwa $700 \mathrm{mg} / \mathrm{l} \mathrm{ab}-$ leiten:

1. $1 \mathrm{mg}$ Halothan in dest. Wasser entspricht:

$\mathrm{Q}=\frac{\mathrm{h}_{\mathrm{Pr}}}{\mathrm{h}_{\mathrm{st}}}=0,65$ bzw. $\mathrm{Q} \times 15,4=\mathrm{mg} / 1$ Halothan

2. $1 \mathrm{mg}$ Halothan in Olivenöl entspricht:

$\mathrm{Q}=\frac{\mathrm{h}_{\mathrm{P}_{\mathrm{r}}}}{\mathrm{h}_{\mathrm{St}}}=0,12$ bzw. $\mathrm{Q} \times 8,7=\mathrm{mg} / 1$ Halothan

Zur quantitativen Analyse absoluter Gaskonzentrationen in Gewebeproben mit Hilfe der Dampfraum-Gaschromatographie
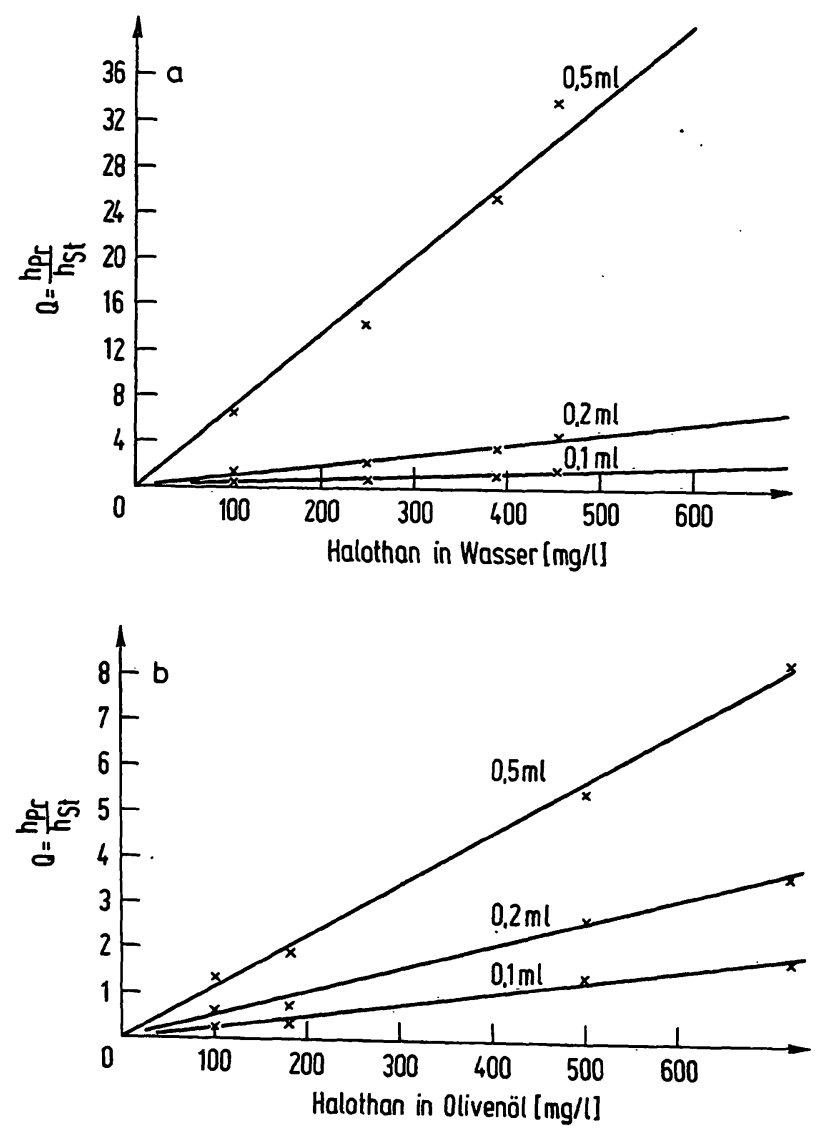

Abb. 1

Chromatographie wäßriger (a) bzw. öliger (b) Lösungen mit eingewogener Halothanmenge. Die Standardkurven zeigen die Beziehung zwischen

$\mathbf{Q}=\frac{h_{P r}}{h_{S t}}\left(h_{\mathbf{P r}}=\right.$ Höhe des Peaks der Probe im Chromatogramm, und absoluter Konzentration in Abhängigkeit vom Lösungsmittel und von der Probenmenge (Angabe an den Kurven) wäre die Herstellung annähernd homogener Lösungen, vergleichbar mit dem Blut, und die Bestimmung des Fettanteils im jeweiligen Gewebe erforderlich. Darauf wurde verzichtet, da als vornehmliches Arbeitsziel die Untersuchung der Dynamik der Konzentrationsabläufe angesehen wurde.

Ausgehend von der Annahme, daß die Löslichkeit des Narkosegases Methoxyfluran ${ }^{2}$ ) mit dem Fettgehalt des jeweiligen Gewebes korrespondiert, haben Eger und Shargel (3) aus dem Verteilungskoeffizienten $\mathrm{Ol} / \mathrm{Gas}$ bei $37^{\circ} \mathrm{C}$ von $825 \pm 58$ einen Koeffizienten Fettgewebe/Gas von $495(825 \cdot 0,60)$ berechnet. Sie sind dabei von einem Fettanteil von $60 \%$ des Volumens des Fettgewebes ausgegangen, der Rest ist im wesentlichen Wasser und Protein. Der Öl/Gas-Verteilungskoeffizient wird für Methoxyfluran mit 970, für Halothan mit 224 und für Äther mit 65 angegeben (4). Die angeführten Úberlegungen und Befunde lassen unserer Meinung nach auch aus den Ergebnissen der DampfraumGaschromatographie Rückschlüsse auf absolute Gaskonzentrationen in Gewebeproben zu, jedoch wird in der folgenden Darstellung auf die Umrechnung verzichtet, zumal die Gewebezusammensetzungen nicht analysiert wurden. Die Konzentrationen werden als Relativiwerte (Q-Werte) angegeben.

Tierversuche

Für die Untersuchungen der Gasverteilung in Geweben wurden 10-12 Wochen alte männliche Albinoratten (Fa. Invanovas, Kisslegg/Allg.) mit einem Körpergewicht von 200-250 g verwendet. Die Tiere wurden in einen abgeschlossenen Käfig gebracht, der von einem Gasgemisch von Lachgas (4 l/min), Sauerstoff $(2 \mathrm{l} / \mathrm{min})$ und Halothan $(1 \% \mathrm{v} / \mathrm{v})$ durchströmt wurde. Nach bestimmten Expositionszeiten wurden die Tiere dekapiert. Das aus den Halsgefäßen abströmende arterio-venöse Mischblut (2 Proben zu je $0,5 \mathrm{ml}$ ) sowie Gewebeproben $(500 \pm 50 \mathrm{mg}$ ) aus verschiedenen Organen (je 2 Proben aus Gehirn, Leber, $M$. quadriceps und dem epididymalen Fettkörper und je eine Probe aus dem Herzmuskel und dem interskapulären Fettkörper) wurden auf ihren Halothangehalt untersucht. Zur Ausbildung eines konstanten Dampfdruckes wurden die Gewebeproben mindestens $40 \mathrm{~min}$ im Wasserbad bei $60^{\circ} \mathrm{C}$ inkubiert.

Zur Untersuchung der Hepatotoxizität lipoidlöslicher Gase bei bestehender Leberverfettung wurde die von IngaLLS et al. (5) beschriebene Variante der Hausmaus (Inzuchtstamm C 57 BL/6 J $\mathrm{ob} / \mathrm{ob}$ ) verwendet, deren homocygote Merkmalsträger durch eine extreme Fettsucht mit Fettleber gekennzeichnet sind. Bei Verteilungsuntersuchungen wurden die Tiere eine Stunde einer Konzentration von $1 \%(\mathrm{v} / \mathrm{v})$ Halothan ausgesetzt. In einer weiteren Versuchsserie zum Nachweis der Leberschädigung wurden obese Mäuse und NMRI-Mäușe an 4 aufeinanderfolgenden Tagen jeweils $1 \mathrm{~h}$ bei $0,5 \%(\mathrm{v} / \mathrm{v})$ Halothan exponiert und am 5. Tage getötet.

In der letztgenannten Versuchsserie wurden von den ${ }^{\text {obesen }}$ Mäusen und den Kontrolltieren histologische Schnitte aus Lebergewebe angefertigt. Die Gasbestimmungen exfolgten bei MäuseVersuchen aus $200 \pm 20 \mathrm{mg}$ Gewebe bzw. 0,2 ml Blut. Lediglich bei Bestimmungen aus der Milz mußte oft bei der geringen Größe des Organs von kleineren Gewebsproben ausgegangen werden.

\section{Ergebnisse}

Durch Untersuchung der Organe nach verșchieden langen Expositionszeiten der Tiere bis zu einer Stunde wurden die in den Abbildungen jeweils in Relation zum Blutspiegel dargestellten Halothan-Konzentrationsverläufe in Leber und Niere (Abb. 2), Herz- und Extremitätenmuskel (Abb. 3) sowie in verschiedenen Fettgeweben und im Gehirn (Abb. 4) bestimmt. Die Konzentrationsangaben für einen bestimmten Zeitpunkt sind jeweils Mittelwerte, die von 5 Tieren ge-

2) Penthrane; 2,2-Dichlor-1,1-difluor-äthyl-methyl-äther. 


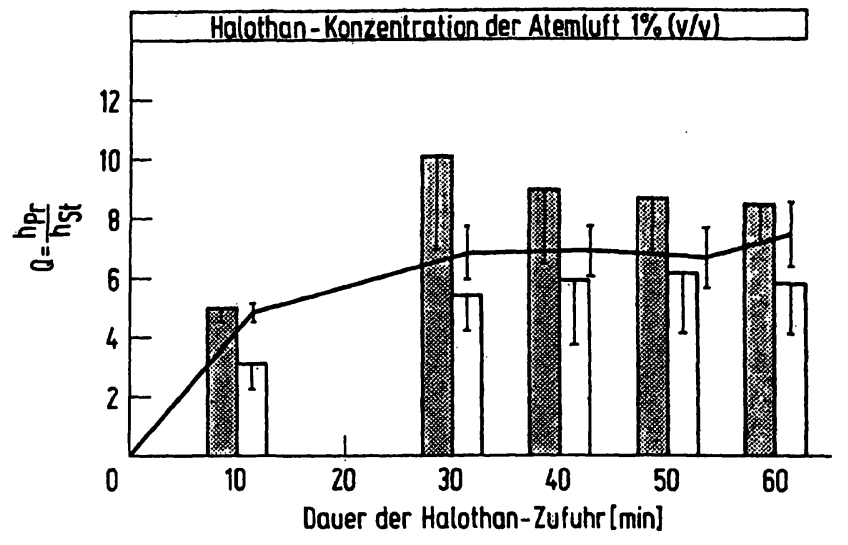

Abb. 2

Verteilung von Halothan in Niere (gerastert), Leber (weiß) und a.-v.Blut (arterio-venöses Mischblut der Halsgefäße, ausgezogene Kurve) bei Exposition der Tiere (Ratten) bis $z u \quad 60 \mathrm{~min}$. Angegeben ist jeweils $\overline{\mathrm{x}} \pm \mathrm{s}(\mathrm{n}=5)$

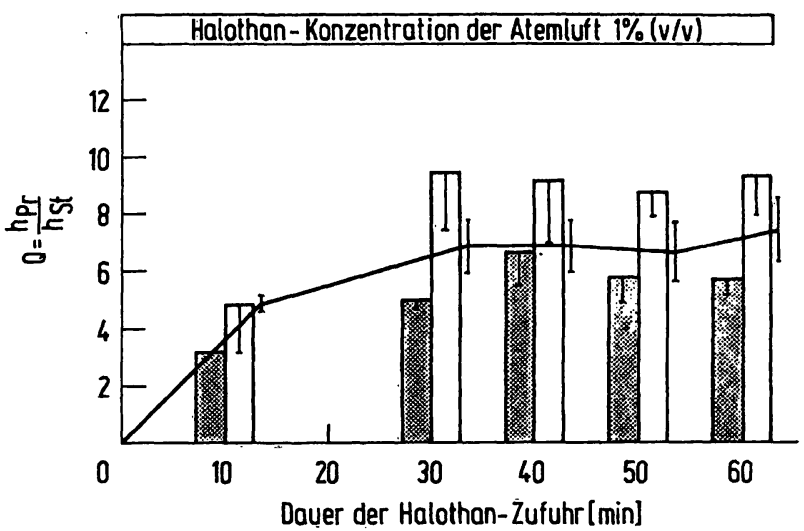

Abb. 3

Anflutung und Verteilung von Halothan im Herzmuskel (weiß) und im Skelettmuskel (gerastert) im Verhältnis zur Konzentration im a.-v.-Blut (ausgezogene Kurve) $\bar{x} \pm s, n=5$. Die Halothan-Konzentration im Herzmuskel der Ratten liegt 30 bis 60 min nach Beginn der Expositionszeit signifikant über der im $M$. quadriceps ( $t$-Test unverbundene Daten; 30,50 und 60 Minutenwert, $p<0,001 ; 40$ Minutenwert $p<0,05$ )

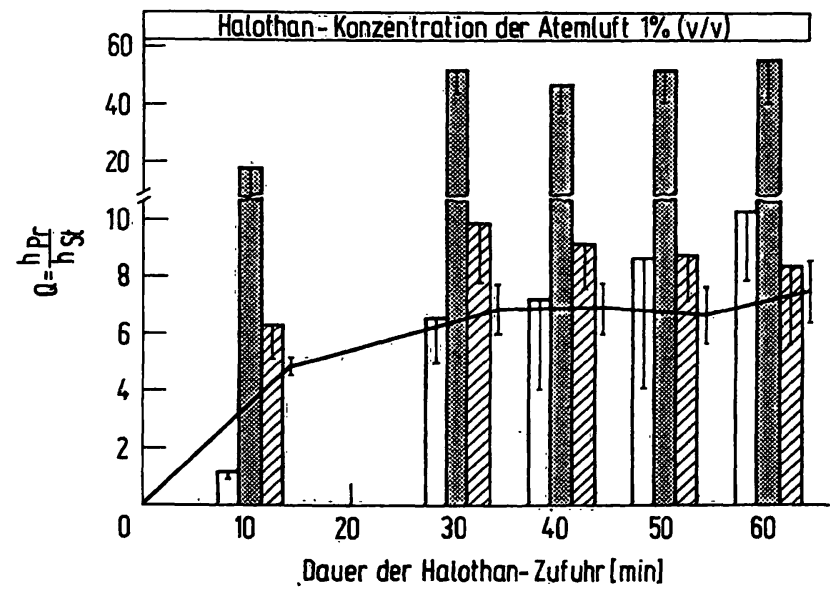

Abb. 4

Anflutung und Verteilung. des lipoidlöslichen Gases (Halothan) in verschiedenen Fettgeweben und im Gehirn (schraffiert) bei Ratten $\bar{x} \pm s, n=5$. Der Konzentrationsanstieg im interscapulären Fettkörper (braunes Fettgewebe, gerastert) erfolgt wesentlich schnelle als im Gewebe des epididymalen Fettkörpers (weißes Depotfett mit geringer Durchblutung (weiß). Ausgezogene Kurve: a.-v.-Blut

wonnen wurden. Unterschiede in den Verteilungsabläufen in einzelnen Geweben treten bei längeren Beobachtungszeiten deutlich hervor (Abb. 5).

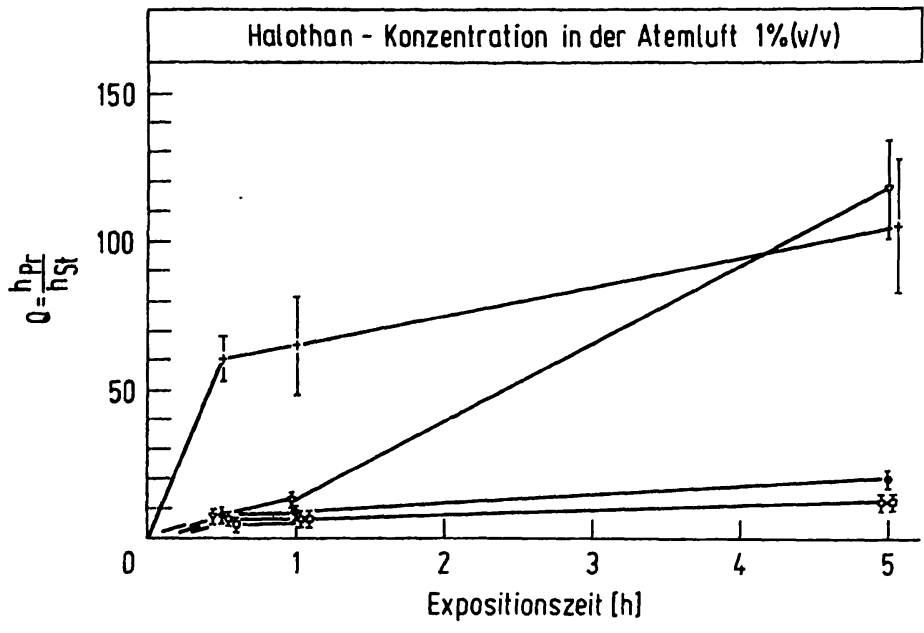

Abb. 5

Halothan-Konzentration in verschiedenen Geweben bei Langzeitexposition der Tiere $(n=5)$. Im stoffwechselaktiven braunen Fettgewebe $(+-+)$ steigt die Konzentration anfangs steil und näher sich dann langsam dem Sättigungswert. Der Anstieg im weißen Depotfett $(\nabla-\nabla)$ erfolgt offenbar kontinuierlich während der ge-

O-O a.-v.-Blut, $\square-\square$ Leber, O-○ M. quadriceps

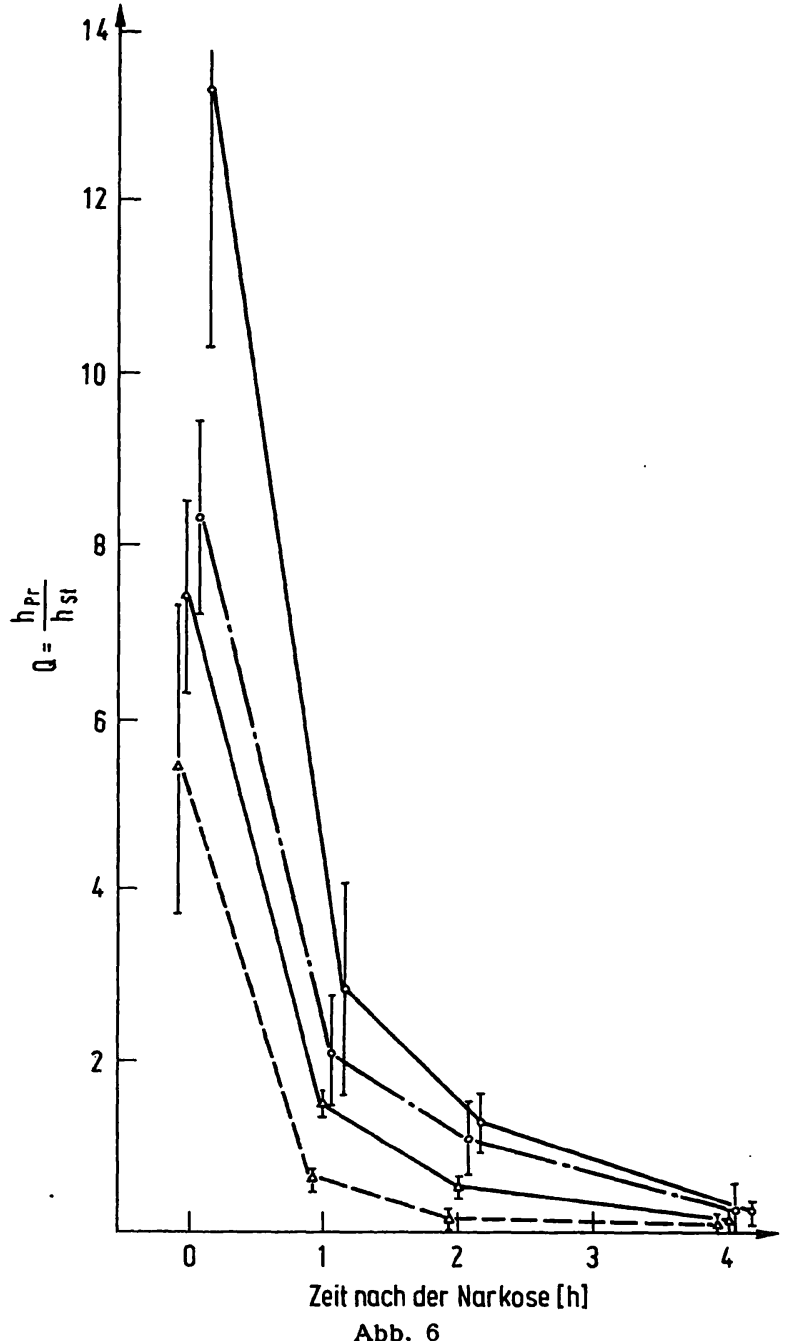

Elimination von Halothan nach der Exposition (1\% v/v, $1 \mathrm{~h}$ ) aus

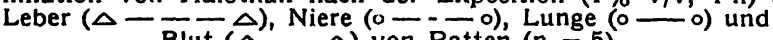

Bei der Gaselimination nach Beendigung der Exposition zeigen die parenchymatösen Organe Leber, Niere und Lunge (Abb. 6), Herz- und Extremitätenmuskel (Abb. 7) 


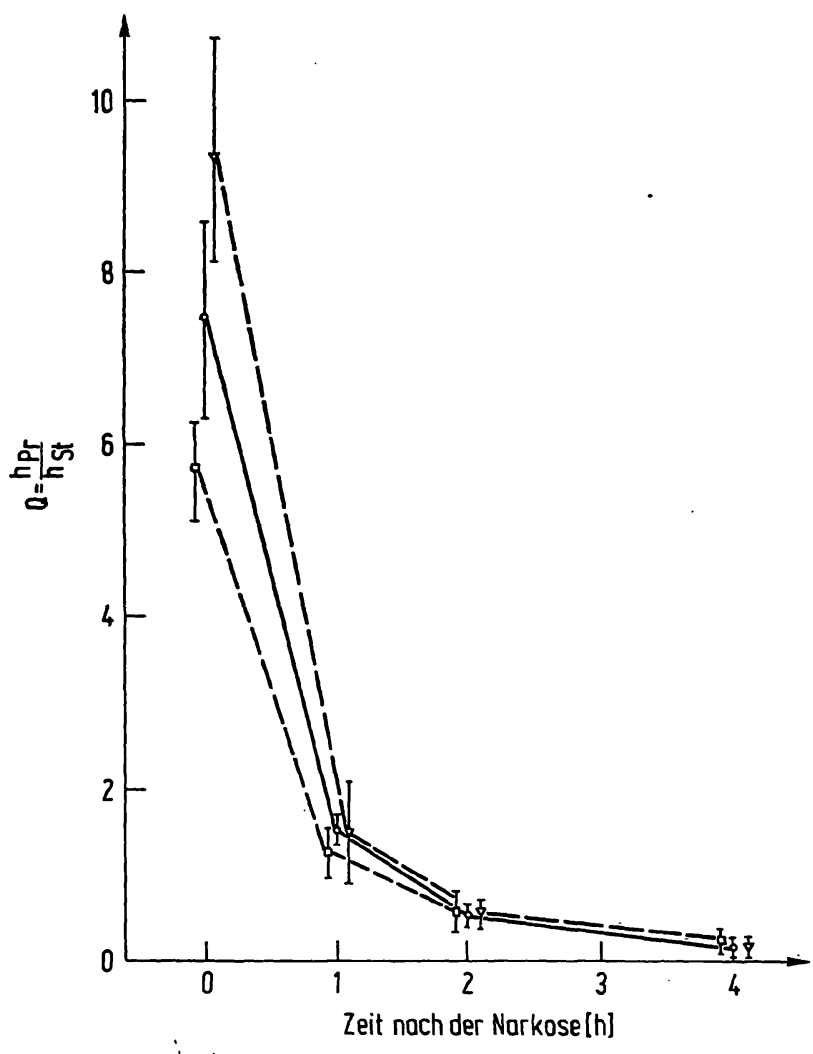

Abb. 7 Halothan-Elimination nach Exposition $(1 \% \mathrm{v} / \mathrm{v}, 1 \mathrm{~h})$, aus Blut $(0)$,
Herzmuskel $(\nabla)$ und quergestreiftem Muskel $(\square)$ von Ratten $(\mathrm{n}=5)$

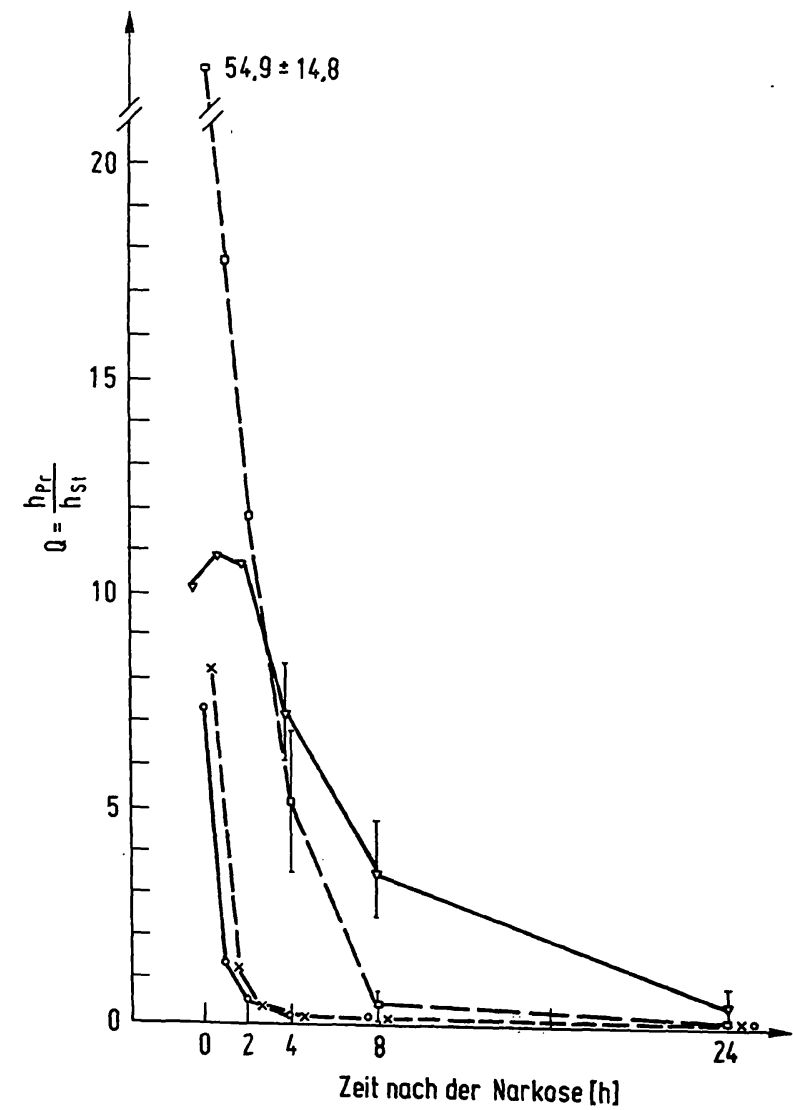

Abb. 8

Elimination von Halothan nach Exposition ( $1 \% \mathrm{v} / \mathrm{v}, 1 \mathrm{~h}$ ) aus braunem $(\square)$ und weißem $(\nabla)$ Fettgewebe im Vergleich zum Konzentrationsabfall im Blut $(O)$ und im Gehirn $(x)$ von Ratten $(n=5)$ und Hirngewebe (Abb. 8) einen exponentiell verlaufenden Konzentrationsabfall mit kurzer Halbwertszeit. Zum Unterschied dazu steigt im weißen Fettgewebe (Abb. 8) die Konzentration im Zuge einer Umverteilung vorübergehend noch an. Das Gewebe nimmt offenbar aufgrund seiner günstigen Lösungsbedingungen noch Halothan aus dem Blut auf.

In den Abbildungen 9 und 10 ist die Halothanverteilung bei obesen Mäusen dargesttellt. Gegenüber den Kontrolltieren ist die Halothankonzentration in der Leber am Ende einer einstündigen Exposition um den Faktor 4 höher. Rückschlüsse von Blutanalysenergebnissen auf Gaskonzentrationen im Lebergewebe sind also nicht ohne Beachtung zusätzlicher Faktoren zulässig.

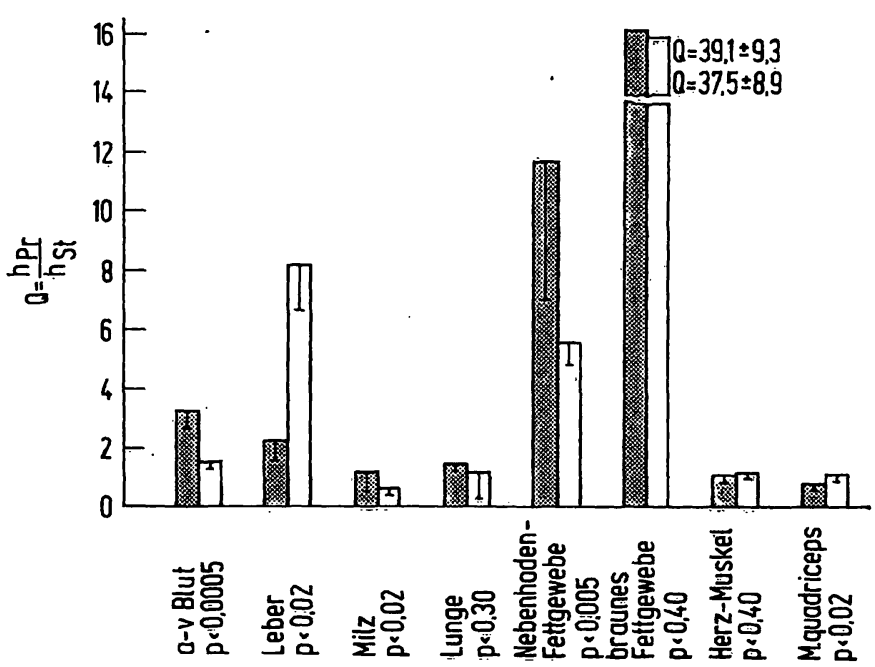

Abb. 9

Einlagerung lipoidlöslicher Gase (Halothan-Exposition $1 \% \mathrm{v} / \mathrm{v} 1 \mathrm{~h}$ ) in verschiedene Gewebe bei normalgewichtigen NMRI-Mäusen (gerastert) $(\mathrm{n}=5)$ und bei Mäusen mit genetisch bedingter Fettsucht Inzuchtstamm C 57 BL $6 \mathrm{~J}$ ob/ob) (weiB) $(n=5)$. Das Körpergewicht der adipösen Mäuse betrug $48+4,5 \mathrm{~g}$ das der normalen Mäuse 8-29 g. In der Fettleber der adipösen Tiere findet sich nach gleicher Expositionszeit $(60 \mathrm{~min})$ eine etwa 4-fach höhere Halothan-Konzentration gegenüber der bei Tieren mit normaler Leber

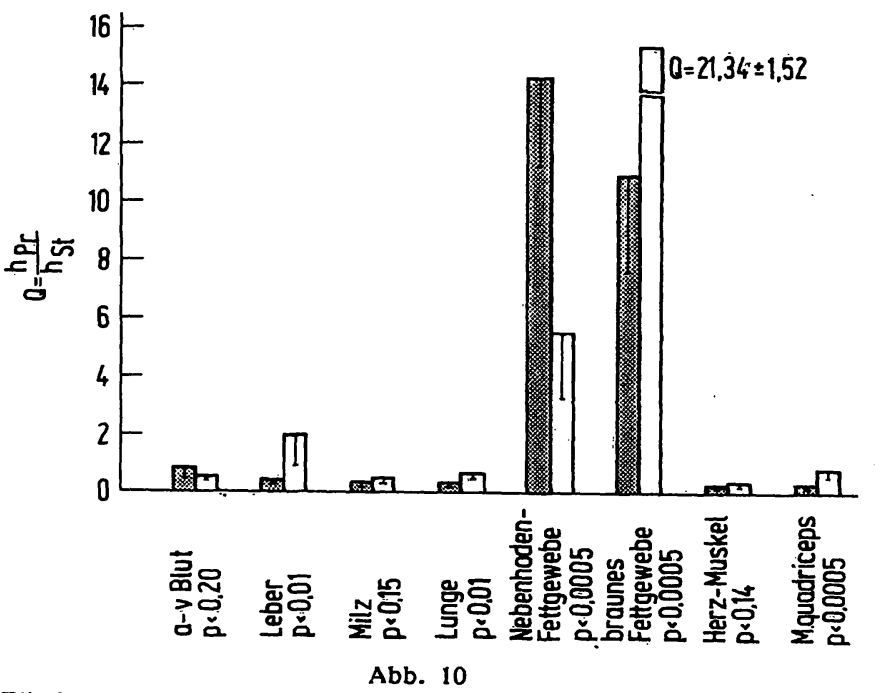
Elimination lipoidlöslicher Gase (30-Minutenwert, Halothanexposition
$1 \% \mathrm{v} / \mathrm{v}, 1 \mathrm{~h})$ bei normalgewichtigen NMRI-Mäusen (gerastert) $(\mathrm{n}=5)$ und bei Mausen mit genetisch bedingter Fettsucht (weiß) $(n=5)$ Die Leber der adipösen Tiere weist auch während der Eliminationszeit eine erhöhte Halothan-Konzentration auf 


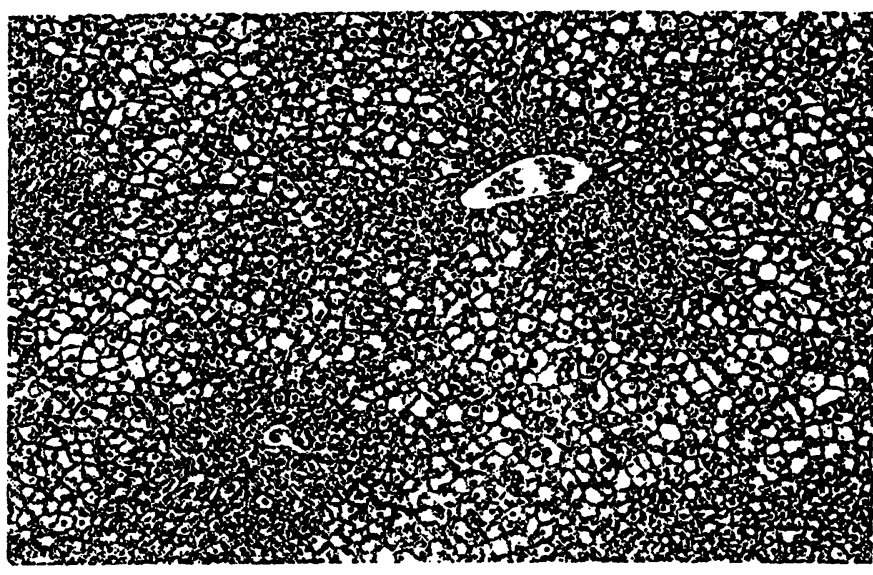

Abb. 11

Leber unbehandelter obeser Mäuse. HE-Färbung, Paraffineinbettung 85 fache Vergrößerung

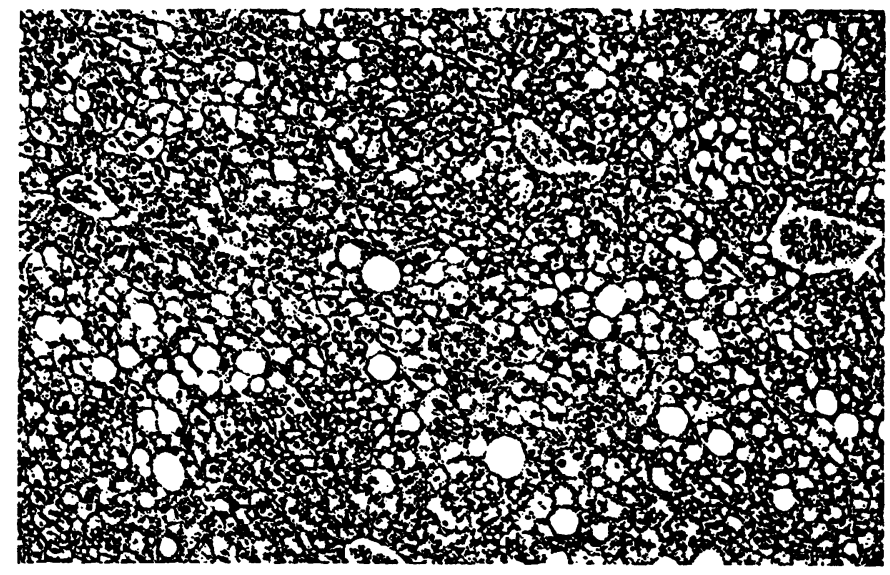

Abb. 12

Diffuse, zum Teil grobblasige Verfettung der Leberzellen nach einstündiger Halothan-Exposition an 4 aufeinanderfolgenden Tagen. Die gelockert. HE-Färbung, Paraffineinbettung, 85 fache Vergrößerung

Die Durchblutung und damit auch die Stoffwechselaktivität des epididymalen Fettgewebes der adipösen Mäuse sind offenbar erheblich vermindert, die Gasaufnahme (Abb. 9) pro Zeiteinheit ist verzögert. Während der Gaselimination (Abb. 10) steigt offenbar die Gaskonzentration im weißen Fettgewebe bei den Tieren beider Versuchsgruppen vorübergehend ebenfalls noch an (vgl. dazu Abb. 8).

Zur Untersuchung der Hepatotoxizität von Halothan bei Fettleber wurden $24 \mathrm{~h}$ nach der 4 . Exposition ( $1 \mathrm{~h}$ $0,5 \%$ (v/v) Halothan pro Tag) lediglich im Fettgewebe obeser Mäuse noch geringe Halothanmengen nachgewiesen, dagegen nicht bei NMRI-Mäusen unter gleichen Versuchsbedingungen. Diese zeigten auch keine histologisch erkennbaren Veränderungen im Lebergewebe. Bei obesen Mäusen war im histologischen Schnitt eine Zunahme der Organverfettung erkennbar (Abb. 11 und 12).

\section{Diskussion}

Bei hibernisierenden Tieren, aber auch bei adulten Ratten und Mäusen sind weißes und braunes Fettgewebe anatomisch und funktionell gut voneinander ab- zugrenzen. Das weiße Fettgewebe hat Depotfunktion, wobei z. B. sein Fettgehalt durch Nahrungsaufnahme und Insulin erhöht wird. Insulinmangel, Fasten u. a. haben einen gegenteiligen Effekt, gleichzeitig sinkt die DNA-Synthese (6).

Das braune Fettgewebe, über das BRÜCK (7) zusammenfassend referierte, weist eine erheblich größere Stoffwechselaktivität als weißes Fettgewebe auf. Die Sauerstoffaufnahme ist pro Einheit Frischgewebe im braunen Fettgewebe $20-50 \mathrm{mal}$ größer als im weißen. In bezug auf die fettfreien Gewebsanteile unterscheidet sich die Stoffwechselaktivität um den Faktor 2-3. Die braune Farbe des interscapulären Fettkörpers beruht auf dem hohen Cytochrom-c-Gehalt und wohl auch auf der hohen Blutfülle des Organs. Braunes Fettgewebe wurde bei verschiedenen neugeborenen Säugern nachgewicsen. Es ist das Organ der sogenannten zitterfreien Thermogenese, welches die postpartal gute Kälteadaption der Neugeborenen durch eine vom Kältezittern unabhängige Wärmebildung bedingt.

Die differente Stoffwechselaktivität und der damit verbundene unterschiedliche Durchblutungsgrad des epididymalen (Depotfett) und des interscapulären (braunes Fett) Fettkörpers sind ursächlich für die pro Zeiteinheit dem Gewebe mit dem Blut zugeführte unterschiedliche Gasmenge (Abb. 4 u. 5). Der Anstieg der Konzentrationskurve des braunen Fettgewebes verläuft anfangs wesentlich steiler und wird bereits zu einer Zeit flacher, nähert sich also dem Sättigungsbereich, in der die Konzentration im weißen Fettgewebe noch linear ansteigt. Für den Menschen wurde unter Berücksichtigung des Lösungsverhaltens von Halothan die für eine 95proz. Blut/Gewebe-Äquilibrierung unter Narkosebedingungen erforderliche Zeit auf $140 \mathrm{~h}$ berechnet, wobei ein Fettanteil des Organismus von $17 \mathrm{l}$ unterstellt wurde (8).

Die Lösungskapazität der Zelle für lipoidlösliche Gase wird von der Relation Fettvolumen/Gesamtvolumen bestimmt. Bei Entwicklung einer Fettsucht nimmt das Volumen der einzelnen Fettzelle erheblich zu. Dabei scheinen bestimmte Stoffwechselleistungen des Gewebes wie maximale Glucoseaufnahme und Stimulierbarkeit der Lipolyse herabgesetzt (zusammenfassend bei Englhardt (9)). Auch die Gewichtszunahme des epididymalen Fettkörpers obeser Mäuse geht mit einer Verminderung der Zellzahl pro Gewichtseinheit einher (10). Die Verlängerung der intrazellulären Diffusionsstrecke bei Volumenzunahme der einzelnen Zelle läßt eine pro Gewichtsanteil verminderte Gasaufnahmerate erwarten.

Die gegenüber normalen NMRI-Mäusen unter gleichen Expositionsbedingungen pro Gewichtseinheit Gewebe verminderte Halothanaufnahme und verzögerte $A b$ gabe im epididymalen Fettkörper obeser Mäuse (Abb. 9 u. 10) hat neben den Veränderungen der Diffusionsbedingungen wahrscheinlich noch einen relativen Blutvolumenmangel als Ursache. Blutvolumenbestimmungen mit ${ }^{51} \mathrm{Cr}$-markierten Erythrocyten ergaben, daß bei 
den homocygoten Merkmalsträgern einem Anstieg des Körpergewichtes auf durchschnittlich $179 \%$ eine $\mathrm{Zu}$ nahme des Blutvolumens auf nur etwa $121 \%$ gegenübersteht (11). Das Gewebe des braunen Fettkörpers scheint von dem Stoffwechseldefekt nicht betroffen. Obese Tiere zeigten gleiche Halothan-Aufnahmeraten wie Normaltiere (Abb. 9).

Entsprechend der Gewebszusammensetzung hinsichtlich ihres Fett- und Wasseranteils können für lipophile Gase bei der Darstellung ihrer Verteilungsabläufe folgende 3 Kompartimente unterschieden werden: Blut, parenchymatöse Organe und Fettgewebe. Die Konzentrationsverteilungen Alveolarluft/Blut und Blut/parenchymatöse Organe nähern sich dabei offenbar schnell einem Gleichgewicht, dessen absolute Konzentrationshöhe vom Anteil des Gases in der Alveolarluft bestimmt wird. Außer von der Expositionszeit des Organismus werden die Konzentrationsverhältnisse im Organgewebe dabei vom Blut/Gewebe-Lösungskoeffizienten bestimmt. Er erreicht für Halothan in verschiedenen Geweben folgende Werte: 1,6 (Niere), 2,6 (Gehirn), 2,6 (Leber), 3,5 (Muskel) und 60 (Fett) (12).

Die Pharmakokinetik und Toxikologie flüchtiger lipoidlöslicher Kohlenwasserstoffe verdient zunehmendes Interesse, da bei steigender Verwendung derartiger Stoffe in Industrie und Haushalt Expositionsgelegenheiten häufiget werden.

Das Schnüffeln (sniffing) verschiedener flüchtiger Kohlenwasserstoffe (Lösungsverdünner, Sprays) zur Herbeiführung eines Rauschzustandes hat in den letzten Jahren bei Jugendlichen Verbreitung gefunden. Jedoch sind Äther-, Chloroform-, Benzin- und Tri(Trichloräthylen)-Sucht bereits länger bekannt.

Im Rahmen toxikologischer Fragestellungen durchgeführte Analysen inkorporierter lipoidlöslicher Gase aus dem Blut können nach den dargelegten Ergebnissen nur geringe Hinweise über tatsächlich im Organismus vorhandene Substanzmengen geben. Gewebsspiegel werden durch solche Analysen nicht erfaßt. Bei Exposition unter bestimmten Gaskonzentrationen erreichte Gewebskonzentrationen werden zudem durch Änderung der Lösungsverhältnisse im Gewebe beeinflußt. Intrazelluläre Fettablagerungen exhöhen die Löslichkeit für lipoidlösliche Gase, das Konzentrationsgleichgewicht zum Blut stellt sich auf einem gegenüber Normalgewebe erhöhten Niveau ein. Der Lipoidanteil am Leberfeuchtgewicht obeser Mäuse, wie sie in den dargelegten Versuchen verwendet wurden, beträgt $19,4 \%$ gegenüber $6,5 \%$ bei Normaltieren (13). Dies bedingt die gaschromatographisch nachweisbare etwa 4 fache Halothan-Konzentrationsüberhồhung im Lebergewebe.
Die Bedeutung der Konzentrationsüberhöhung als pathogenetischer Faktor wird-durch den Vergleich „therapeutischer" Bereiche mit toxisch wirksamen Konzentrationen verdeutlicht. Die einmalige $G$ abe von 0,8 bis $1,0 \%$ (v/v) Halothan in der Atemluft führt bei Ratten nur zu geringfügigen Fermentaktivitätssteigerungen im Blut. Diese treten nach 4-stündiger Narkose mit $1,5 \%(\mathrm{v} / \mathrm{v})$ Halothan deutlicher hervor, bei Steigerung auf $2,0 \%(v / v)$ werden bereits die ersten Todesfälle beobachtet, bei $2,5 \%(\mathrm{v} / \mathrm{v})$ überleben nur noch wenige Tiere (14). Danach erscheint der Schluß zulässig, daß die nach Mehrfachexposition bei 0,5 (v/v)\% Halothan in der Atemluft lichtmikroskopisch nachweisbare $\mathrm{Zu}-$ nahme der Leberzellverfettung (Abb. 11 u. 12) durch Erreichen ,toxischer" Konzentrationen in der Leber bei niedriger Dosierung in der Atemluft bedingt wurde.

Auch wenn hepatotoxische Effekte nach einmaliger Halothannarkose unter Einhaltung geeigneter Dosierungen nicht bewiesen sind, so muß doch beim $\mathrm{Zu}$ sammentreffen mit zusätzlichen Noxen wie präoperativem Leberschaden, Virushepatitis, Hypoxie, Hypotonie, Verabfolgung von Medikamenten, Bluttransfusionen, Oberbauchtraumen oder schweren Infektionen mit diskreten oder massiven Leberzellschädigungen gerechnet werden (15). Der dargelegte Mechanismus einer Konzentrationsüberhöhung bei pathologischen Fettablagerungen in der Leberzelle, trotz "normaler" Dosierung des Gases in der Atemluft, kann zumindest bei einigen der angeführten Noxen ursächlich sein. Die Befunde unterstreichen die Forderung nach präoperativer, zumindest orientierender Leberdiagnostik (16) und nach sorgfältiger Auswahl des geeigneten Narkoseverfahrens.

Zur Verhinderung von Gesundheitsschäden durch chronische Gaseinatmung werden im Rahmen der Arbeitsmedizin maximale Arbeitsplatzkonzentrationen (MAK-Werte) angegeben. Der MAK-Wert besagt, da von der angegebenen Konzentration nach den bisherigen Erfahrungen beim Umgang mit solchen Stoffen oder/und aufgrund langdauernder Tierversuche angenommen werden kann, daß selbst bei 8-stündiger Einwirkung diese im allgemeinen keine Schädigung auf die Gesundheit haben. Berücksichtigt man die dargelegten Verteilungsänderungen von Gasen bei Leberverfettung, so ergibt sich für die arbeitsmedizinische Begutachtung, daß neben der Einhaltung der MAKWerte auch individuelle Dispositionen zu berücksichtigen sind.

\section{Danksagung}

Frl. Seewald, Frl. Bäumler, Hetrn Reich und Herrn Müller bin ich für die technische Hilfe bei der Durchführung der Versuche zu Dank verpflichtet. 


\section{Literatur}

1. Gostomzyk, J. G. (1971), Anaesthesist 20, 212-215. - 2. 355. - 9. Englhardt, A. (1972), Deut. Med. Wochenschr. GostomzyK, J. G. \& Streffer, C. (1969), Blutalkohol 6, 211-224. 97, 161-166. - 10. Hellmann, B., Thelander, L. \& Täljedal, 3. Eger, E. \& Sharged, R. (1963), Anesthesiology 24, 625-627. J. (1963), Acta Anat. 55, 286-294. - 11. Yen, T. T., Steinmetz, 4. Saidman, L. J., Eger, E. J., Munson, E. S., Babad, A. A. \& J. \& Simpson, P. J. (1970), Proc. Soc. Exp. Biol. Med. 133, Mallem, M. (1967), Anesthesiology 28, 994-1002. - 5. Ingalls, 307-308. - 12. Eger, E. I. \& Larson, C. P. jr. (1964), Brit. J. A. M., Drckie, M. M. \& Sneld, G. D. (1950), J. Heredity 41, Anesth. 36, 140-149. - 13. Grimmel, K., Beneke, G., Brauch317-318. - 6. Hollenderg, C. H., ANGel, A. \& Stemer, G. (1970), Can. Med. Ass. J. 103, 843-849. - 7. BRück, K. (1967), Naturwissenschaften 54, 156-162. - 8. LARSON, C. P., EGER, E. J. \& Severinghaus, J. W. (1967), Anesthesiology 23, 349 bis LE, J. \& Rakow, L. (1963), Beitr. Pathol. 142, 275-305. 14. Clauberg, G. (1970), Anaesthesist 19, 317-324. - 15 Pichlmayr, J. \& Picirlmayr, R. (1964), Anaesthesist 13, 293-302. 16. FODOR, L. (1970), Med. Welt 21/26, 2005-2009.

Prof. Dr. J. G. Gostomzyk

Department für Anaesthesiologie der Universität Ulm 7900 Ulm (Donau)

Steinhövelstraßc 9 